\title{
SYNOPSIS OF THE GENUS ALYSSUM IN IRAN
}

\author{
K. Kavousi ${ }^{1}$, Z. NAZARI ${ }^{2,3}$ and H. Ejtehadi ${ }^{2}$ \\ ${ }^{1}$ Department of Biology, Science and Research Branch, Islamic Azad University, Tehran, Iran \\ E-mail: KKavorsia697@gmail.com \\ ${ }^{2}$ Department of Biology, Faculty of Science, Ferdowsi University of Mashhad \\ Azadi square, Mashhad, 9177948974, Iran \\ E-mails: nazary.zahra@gmail.com, Hejtehadi@um.ac.ir \\ ${ }^{3}$ Agricultural and Natural Resources Research Center of Semnan Province (Shahrood) \\ Shahrood, Semnan Province, Iran
}

(Received 17 August, 2014; Accepted 5 November, 2014)

\begin{abstract}
A complete, up to date checklist of Alyssum species reported from Iran is presented in this review. The distribution of these species was considered in Iran and in the adjacent countries, too. Additional records were obtained from Flora Iranica, Flora of Turkey and other references. In Iran some species (e. g., Alyssum hezarmasjedensis, A. mozaffarianii, A. persicum, A. polycladum, A. stipitatum, A. turgidum) have very restricted distribution. Iran is the second important locality for the following species: A. anatolicum, A. contemptum, $A$. filiforme, A. iranicum, A. lycaonicum, A. niveum, A. penjwinense. The other Alyssum species are widespread in Iran and in the adjacent countries.
\end{abstract}

Key words: Alyssum, biodiversity, distribution, Iran

\section{INTRODUCTION}

The genus Alyssum L. includes about 230 species around the world (AlShehbaz et al. 2006, Bailey et al. 2006, Persson 1971). The accepted list and literature review of genus Alyssum L. available in the IPNI database contain 187 species worldwide. In Flora Iranica 31 Alyssum species were reported from Iran by Rechinger (1968). Recently (since 1990) the number of Iranian Alyssum species has been increased considerably by Kavousi and his coworkers. Currently 53 Alyssum species are known from Iran, which are classifying in 5 sections (Kavousi et al. 2010, 2011, Kavousi and Nazari 2014). Several new species have been described directly from Iran, such as Alyssum mozaffarianii Kavousi, A. stipitatum Kavousi et T. R. Dudley and A. hezarmasjedensis Kavousi et Naza- 
ri. The other 19 species are contributed in Flora Iranica. A complete list of the Iranian Alyssum species and an overview on their distribution in Iran and in other adjacent localities are given in this study.

\section{MATERIALS AND METHODS}

All known localities of Alyssum in Iran were studied according to the information obtained from the publications and from herbarium specimens in the following Iranian herbaria: Institute of Forest and Rangelands, Shahid Beheshti University, Isfahan University, Evin, TUH, and FUMH. Specimen records were also checked in the following foreign herbaria: Vienna, St-Petersburg, Ankara, Ghazi, Hacceteppe, Hungary, Tbilisi herbaria.

Furthermore, references as Baumgartner (1909), Boissier (1867), Davis (1965), Dudley $(1964 a, b, 1965,1968)$, Duman and Aytaç (2000), Kavousi (2001), Kavousi et al. (2010, 2011), Nazari et al. (2013), Nyárády (1927, 1929, 1949), Orcan (1999), Orcan and Binzet (2006), and Orcan and Misırdalı (1998) have been used and considered for literature review.

\section{DISCUSSION}

According to Dudley $(1964 a, b)$, the Alyssum genus has 6 sections worldwide and 5 of them have been recognised in Iran as well. The section Tetradenia including three perennial and spinose species, i.e. A. cochleatum, A. lapeyrousianum and $A$. spinosum, is known from North Africa, the Mediterranean Europe and the Pyrenees. None of these three species has been found and reported from Iran up to date.

The number of species in the sections Psilonema, Meniocus, Alyssum, Gamosepalum and Odontarrhena in Iran are 3, 3, 16, 11 and 20 species, respectively.

An overview is presented below with short descriptions of all Alyssum sections, full list of species in each sections and the distribution of the species in Iran and other countries.

\section{Section Meniocus (Desv.) Hook. in Benth. and Hook., Gen. Plant. 1: 74 (1862)}

Plant annual, long filaments unilaterally winged and toothed. Short filaments unilaterally winged and toothed. Short filaments with free, or basically connate appendages. Nectaries globose. Silicles dehiscent, glabrous, or with indumentums or simple seta (rarely forked), frequently papillose, locules $2-4(-8)$, ovulate with distinctly lateral placentation; valves always compressed. Seeds winged or wingless, mucilaginous. 
Alyssum heterotrichum Boiss., Diagn. Pl. Orient., ser. 1, 6: 15 (1845). - Iran, Kazakhstan.

Alyssum linifolium Steph. ex Willd., Spec. Plant., ed. 4, 3(1): 467 (1800). Iran, Turkey, Europe, North Africa, Caucasus, Siberia, India, Australia, Levant. Alyssum meniocoides Boiss., Ann. Sci. Nat., Bot. sér. 2, Paris, 17: 158 (1842). - Afghanistan, Iran.

Section Psilonema (Meyer) Hook. in Benth. and Hook., Gen. Plant. 1: 74 (1862)

Plants annual. Filaments always slender, wingless, edentate, and unappendaged. Nectaries erect and peg-like or globose, silicles dehiscent, glabrous, or with monomorphic or dimorphic indumentums, held in the same direction as pedicels and with biovulate locules; valves equally inflated. Seeds winged or wingless, mucilaginous.

Alyssum alyssoides (L.) L., Syst. Nat., ed. 10, 2: 1130 (1759). - Europe, North Africa, East India, USA, Canada, Argentina, Iran.

Alyssum dasycarpum Steph. ex Willd., Spec. Plant., ed. 4, 3(1): 467 (1800). Europe, Levant, Caucasia, East India.

Alyssum homalocarpum (Fish. et Meyer) Boiss., Fl. Orient. 1: 285 (1867). Egypt, Arabia, Iraq, Iran, Pakistan, Syria, Jordan, Palestine.

\section{Section Alyssum L. Boiss., Fl. Orient. 1: 264, 274 (1867)}

Plants annual, biennial, or if perennial then with sterile shoots and winter rosettes. Sepals monomorphic, free, but often persistent in fruit; inner surface always glabrous. Petals predominantly yellow, occasionally pale, and rarely whitish. Filaments free, unilaterally or bilaterally winged, and/or dentate, and/or appendaged. Nectaries globose, glabrous, or with monomorphic or wingless, mucilaginous.

Alyssum aizoides Boiss., Ann. Sci. Nat., Bot. sér. 2, Paris, 17: 153 (1842). Turkey, Iran.

Alyssum contemptum Schott et Kotschy, Oesterr. Bot. Wochenbl. 4(22): 177 (1854). - Turkey, Levant, Iran.

Alyssum desertorum Stapf, Denkschr. Akad. Wien, Math. Naturw. 51: 33 (1886). - Europe, Asia, USA.

Alyssum iranicum Hausskn. ex Baumg., Jahresb. Landes-Gymn. Baden bei Wien 48: 9 (1911). - Levant, Turkey, Iran.

Alyssum lanceolatum Baumg., Jahresb. Landes-Gymn. Baden bei Wien 48: 11 (1911) - Iran, Afghanistan. 
Alyssum marginatum Steud. ex Boiss., Ann. Sci. Nat., Bot. sér. 2, Paris, 17: 157 (1842). - Levant, Turkey, Russia, India.

Alyssum minus (L.) Rothm., Repert. Spec. Nov. Regni Veg. 50: 77 (1941). Europe, North Africa, Asia.

Alyssum minutum Schlecht ex DC., Syst. Nat. 2: 319 (1821). - Southeastern and Eastern Europe, North Africa, Crete, Cyperus, Aegean Island, Turkey, Syria, Iran.

Alyssum muellerii Boiss. et Buhse, Nouv. Mém. Soc. Imp. Nat. Moscou 12: 16 (1860). - Russia, Armenia, Caucasus, Northern Iran.

Alyssum persicum Boiss., Ann. Sci. Nat., Bot. sér. 2, Paris, 17: 152 (1842). Iran.

Alyssum repens Baumg., Enum. Stirp.Transsilv. 2: 237 (1816). - Europe, Levant, Caucasia, Iran.

Alyssum stapfii Vierh., Verh. zool.-bot. Ges. Wien 64: 260. t. 7, fig. 1 (1916). - Turkey, Levant, Afghanistan, Iran.

Alyssum strictum Willd., Spec. Plant., ed. 4, 3(1): 464 (1800). - Levant, Caucasia, Iran.

Alyssum strigosum Banks et Soland., in Russell, Nat. Hist. Aleppo, ed. 2, 2: 257 (1794). - Europe, North Africa, Levant, Iran, Afghanistan.

Alyssum szovitsianum Fisch. et C. A. Mey., Index Semin. Hort. Petrop. 4: 31 (1837). - Levant, India, Iran.

Alyssum turkestanicum Regel et Schmalh., in Regel, Descr. Pl. Nov. Rar. Fedtsch. 6. (1882). - Transcaspia, Iran, Afghanistan.

\section{Section Gamosepalum (Hausskn.) T. R. Dudley,} J. Arnold. Arbor. 45: 70 (1964)

Plants perennial, with sterile shoots. Sepals distinctly dimorphic, always persistent, and often inflated in fruit, appearing connate because of interlocking indumentum, inner surfaces with indumentum. Petal whitish with purple venation at limb bases, or yellow, long filaments bilaterally winged, edentate and appendaged connate, or if free then contiguous with overlapping wings, short filaments with basal, connate appendages or teeth. Silicles dehiscent. Always with indumentum, held in the same direction as pedicles, and with biovulate locules, valves equally or unequally inflated. Seeds narrowly winged or wingless, mucilaginous.

Alyssum baumgartnerianum Bornm. ex Baumg., Jahresb. Landes-Gymn. Baden bei Wien 48: 6. (1911). - Turkey, Syria, Lebanon, Palestine, Iran.

Alyssum corningii T. R. Dudley, J. Arnold Arb. 45: 72, figs 1-11 (1964). Turkey. Iran. 
Alyssum harputicum T. R. Dudley, J. Arnold Arb. 45: 76, pl. IV, figs 1-12 (1964). - Turkey, Iran.

Alyssum hezarmasjedensis Kavousi et Nazari, Novon, 23(1): 59 (2014). - Iran.

Alyssum lepidoto-stellatum (Hausskn. et Bornm.) T. R. Dudley, J. Arnold Arbor. 45: 70 (1964). - Turkey, Iran.

Alyssum lycaonicum (O. E. Schulz) T. R. Dudley, J. Arnold Arb. 45: 79. Turkey, Iran.

Alyssum niveum T. R. Dudley, J. Arnold Arb. 45: 78, pl. III, fig. 12 (1964). Turkey, Iran.

Alyssum paphlagonicum (Hausskn.) T. R. Dudley, J. Arnold Arb. 45: 71 (1964). - Turkey, Iran.

Alyssum sulphureum T. R. Dudley et Hub.-Mor., J. Arnold Arb. 45: 74, pl. IV, figs 13-26 (1964). - Turkey, Syria, Iraq.

Alyssum tetrastemon Boiss., Ann. Sci. Nat., Bot. sér. 2, Paris, 17: 153 (1842). Turkey, Iran.

Alyssum thymops (Hub.-Mor. et Reese) T. R. Dudley, J. Arnold Arb. 45: 71 (1964). - Turkey, Iran.

Section Odontarrhena (Meyer) Koch, Synops. Fl. Germ. Helv. p. 59 (1835).

Plants perennial, with sterile shoots and winter rosettes, rarely biennial. Pedicels rigid, divergent and spreading, or slender, sigmoid and deflexed. Petals yellow, usually obovate, rarely with basal appendages. Long filaments free, always unilaterally or bilaterally winged, and variously dentate. Short filaments always with free or connate appendages, or teeth. Silicles dehiscent or indehiscent, glabrous or with indumentum, or rarely papillose, held in the same direction as pedicels or pendulous, and with uniovulate locules; valves equally or unequally inflated, or compressed and undulate (samaroid and papyraceous in subsect. Samarifera). Seeds winged or wingless, rarely mucilaginous.

Alyssum anatolicum Hausskn. ex Nyár., Bul. Grad. Bot. Univ. Cluj 9: 40, t. 5, fig. 111; t. 6, fig. 46; t. 7, fig. 56; t. 9, figs 62-63; pl. 29 (1929). - Turkey, Iran. Alyssum argenteum All., Misc. Taur. 5: 73 (1774). - Italy, Iran, Balkans.

Alyssum bracteatum Boiss. et Buhse, Nouv. Mem. Soc. Imp. Nat. Moscou 12: 18 (1860). - Iran, Caucasia. Iran.

Alyssum callichroum Boiss., Diagn. Pl. Orient., sér. 2, 5: 34 (1856). - Turkey,

Alyssum condensatum Boiss. et Hausskn. ex Boiss., Fl. Orient. 1: 268 (1867). - Iran, Turkey, Syria, Lebanon, Iraq. 
Alyssum constellatum Boiss., Ann. Sci. Nat., Bot. sér. 4, Paris, 2: 244 (1854). - Iran, Turkey, Iraq.

Alyssum filiforme Nyár., Bul. Grad. Bot. Univ. Cluj 9: 35, t. 5, fig. 79; t. 6, fig. 40; t. 7, fig. 49; t. 10, fig. 53 (1929). - Iran, Turkey.

Alyssum inflatum Nyár., Bul. Grad. Bot. Univ. Cluj 9: 43, t. 5, fig. 113; t. 6, fig. 41; t. 7, figs 50-51; t. 9, figs 54-58 (1929). - Iran, Transcaspian.

Alyssum lanigerum DC., Syst. Nat. 2: 308 (1821). - Iraq, east to Afghanistan. Alyssum longistylum Grossh., in Grossheim and Schischk. Scheda Herb. Pl. Or. Exsicc. 18 (1924). - Iran, Armenia, Caucasia, Crimea.

Alyssum mozaffarianii Kavousi, Iranian J. Bot. 9(1): 48 (2001). - Iran.

Alyssum murale Waldst. et Kit., Descr. Icon. Pl. Rar. Hung. 1: 5, t. 6 (1799). - Europe, Levant, Iran.

Alyssum obtusifolium Steven ex DC., Syst. Nat. 2: 305 (1821). - Iran, Russia, Turkey.

Alyssum penjwinense T. R. Dudley, Notes Roy. Bot. Gard. Edinburgh 24: 162, pl. 7 , fig. 4 (1962). - Iran, Iraq.

Alyssum polycladum Rech. f., Phyton 3: 55 (1951). - Iran.

Alyssum sibiricum Willd., Spec. Plant., ed. 4, 3(1): 465 (1800). - Iran, Balkans, Turkey, Crimea, Caucasia.

Alyssum singarense Boiss. et Hausskn. ex Boiss., Fl. Orient. Suppl. 49. (1888). - Iran, Iraq.

Alyssum stipitatum Kavousi et T. R. Dudley, Iranian J. Bot. 9(1): 48. - Iran.

Alyssum tortuosum Waldst. et Kit., Descr. Icon. Pl. Rar. Hung. 1: 94, t. 91 (1799). - Iran, Europe to Siberia.

Alyssum turgidum T. R. Dudley, Great Basin Naturalist 24(1): 7, pl. I, figs A-E, G-K (1964). - Iran.

\section{RESULTS}

Boissier (1867) in Flora orientalis presented 14 Alyssum species for the first time from Iran (Persia) as A. bracteatum, A. lanigerum, A. minimum, $A$. persicum, A. bungei, A. argenteum, A. hirsutum, A. campestre, A. szovitsianum, $A$. marginatum, A. heterotrichum, A. linifolium, A. dasycarpum and $A$. murale. The above list was completed in Flora Iranica (T. R. Dudley in Rechinger 1968) with Alyssum homalocarpum, A. contemptum, A. strictum, A. alyssoides, A. meniocoides, A. desertorum, A. stapfii, A. repens, A. iranicum, A. lanceolatum, A. baumgartnerianum, A. constellatum, A. longistylum, A. polycladum, A. inflatum, and A. turgidum.

In recent years Alyssum mozaffarianii, A. stipitatum, A. callichroum, A. sibiricum, $A$. condensatum, $A$. harputicum, and $A$. obtusifolium were reported by Kavousi (2001), Alyssum tetrastemon, A. paphlagonicum, A. niveum, A. sulphure- 
um, A lepidoto-stellatum, A. corningii, and A. thymops by Kavousi et al. (2010); and A. anatolicum and A. filiforme by Kavousi et al. (2011). A new species, Alyssum hezarmasjedensis was described recently from Iran by Kavousi and Nazari (2014).

In our recent investigations at Khorassan Mts; Esfarayen, Saluk, ShahJahan and Shahvar Peak, A. aizoides and A. tortuosum have been reported for the first time from Iran (Nazari et al. 2013).

Alyssum aizoides is only known from Turkey. Alyssum tortuosum, known with a wide distribution from eastern Europe and Siberia, was synonymised erroneously with $A$. inflatum (Akhani 2003), since there are a lot of differences between the two species.

Currently 53 Alyssum species are known from Iran and it seems that Iran is the centre for endemism of this genus. More field investigations are necessary to reveal more information on Iranian Alyssum.

Iran is very rich in Alyssum taxa. Several species have been reported for the first time from Iran, from those special areas with species forming a principle vegetation communities special in central Alborse and eastern Alborse in north east Khorassan.

\section{REFERENCES}

Akhani, H. (2003): Note on the flora of Iran 4. Two new records and synopsis of the new data on Iranian Cruciferae since flora Iranica. - Candollea 58: 369-385.

Al-Shehbaz, I. A., Beilstein, M. A. and Kellogg, E. A. (2006): Systematics and phylogeny of the Brassicaceae: an overview. - Plant Syst. Evol. 259: 89-120. http://dx.doi. org/10.1007/s00606-006-0415-z

Bailey, C. D., Koch, M. A., Mayer, M., Mummenhoff, K., O’Kane, S. L. jr., Warwick, S. I., Windham, M. D. and Al-Shehbaz, I. A. (2006): Toward a global phylogeny of the Brassicaceae. - Mol. Biol. Evol. 23: 2145-2160. http://dx.doi.org/10.1093/molbev/msl087

Baumgartner, G. (1909): Die ausdauernden Arten der Sectio Eualyssum, aus der Gattung Alyssum. III. - Jahresber. nieder-öst. Landes-Lehrersem., Wiener-Neustadt, 34: 1-32.

Boissier, E. (1867): Flora Orientalis: sive, Enumeratio plantarum in Oriente a Graecia et Aegypto ad Indiae fines hucusque observatarum. Vol. 1. - H. Georg, Basilieae, pp. 263-287. http:// dx.doi.org/10.5962/bhl.title.20323

Davis, P. H. (ed.) (1965): Flora of Turkey and the East Aegean Islands. Vol. 1. - Edinburgh University Press, Edinburgh.

Dudley, T. R. (1964a): Studies in Alyssum; near eastern representatives and their allies. - J. Arnold Arbor. 45: 57-100.

Dudley, T. R. (1964b): Synopsis of the Genus Alyssum. - J. Arnold Arbor. 45: 358-373.

Dudley, T. R. (1965): Alyssum L. - In: Davis, P. H. (ed.): Flora of Turkey and the East Aegean Island. 1. Edinburgh University Press, Edinburgh, pp. 362-406.

Dudley, T. R. (1968): Alyssum L. - In: Rechinger K. H. (ed.): Flora Iranica, Cruciferae. 58/28. Akademische Druck- u. Verlagsanstalt, Graz (Austria), pp. 146-170. 
Duman, H. and Aytaç, Z. (2000): Alyssum nezaketiae new species from central Anatolica. - Israel J. Plant Sci. 48(4): 317-319. http://dx.doi.org/10.1092/CRFR-Q6P3-BQ6H-C99V

Kavousi, K. (2001): Notes on the plant family Cruciferae in Iran, new taxa and new records. - Iranian J. Bot. 9(1): 47-57.

Kavousi, K. and Nazari, Z. (2014): A new species of Alyssum (Brassicaceae) from northeastern Iran. - Novon 23: 59-61. http://dx.doi.org/10.3417/2010116

Kavousi, K., Duman, H., Nazari, Z. and Joharchi, M. R. (2010): Additions to the flora of Iran. - Kanitzia 17: 29-31.

Kavousi, K., Duman, H., Nazari, Z. and Jouharchi, M. R. (2011): The genus Alyssum section Gamosepalum in Iran. - Acta Bot. Hung. 53(1): 145-149. http://dx.doi.org/10.1556/ ABot.53.2011.1-2.e1

Nazari, Z., Ejtehadi, H. and Kavousi, K. (2013): Taxonomy of Alyssum sect. Gamosepalum on the basis of anatomical properties. - Acta Bot. Hung. 56(1-2): 179-184. http:// dx.doi.org/10.1556/ABot.56.2014.1-2.13

Nyárády, E. (1927): Studiu preliminar, asupra unor specii de Alyssum din sectia Odontarrhena. - Bul. Grad. Bot. Univ. Cluj 7: 1-29.

Nyárády, E. J. (1929): Vorstudium über einige Arten der Sectio Odontarrhena der Gattung Alyssum. - Bul. Grad. Bot. Univ. Cluj 9: 1-68.

Nyárády, E. J. (1949): Synopsis specierum, variationum et formatum sectionis Odontarrhenae Generis Alyssum. - Anal. Acad. Repub. Pop. Romane A. 3: 1-130.

Orcan, N. (1999): A new Alyssum record for the flora of Turkey. - Israel J. Plant Sci. 47(4): 291-294. http://dx.doi.org/10.1080/07929978.1999.10676787

Orcan, N. and Binzet, R. (2006): A new record for the Flora of Turkey, Alyssum idaeum Boiss. \& Heldr. - Pakistan J. Bot. 38(4): 931-933.

Orcan, N. and Misirdali, H. (1998): A new Alyssum for the Flora of Turkey. - Coll. Bot. (Barcelona) 23: 142-144.

Persson, J. (1971): Studies in the Aegean flora, 19. Note on the Alyssum and some other genera of Cruciferae. - Bot. Notiser 124(3): 339-418.

Rechinger, K. H. (ed.). (1968): Flora Iranica. Vol. 57. - Akademische Druck- u. Verlagsanstalt, Graz. 